State), where he specializes in modeling electronic control systems for mechanical systems and leads the Mechatronics Research Laboratory. Wang is a Distinguished Professor of Mechanical Engineering and professor of materials science and engineering also at Penn State, and his research interests focus on the transport, materials, manufacturing, and modeling aspects of batteries and fuel cells. Rahn and Wang co-direct Penn State's Battery and Energy Storage Technology (BEST) Center.

This book briefly tours the electrochemistry of batteries and then follows with mathematical methods for modeling such electrochemical systems. The models are built up from the fundamental processes in the batteries. The governing equations are presented for rechargeable batteries, based mainly on thermodynamics, reaction kinetics, and transport in porous electrodes and electrolyte, plus the impact of cell voltage, temperature, and aging. From there, the authors present 40 pages of discretization methods. Much of the same mathematical approaches are used in the chapter on system response. The chapter on battery systems models attempts to give fair balance to the several approaches, making clear that there are numerous ways to model the batteries and that estimation methods are still necessary. The last chapter is dedicated to battery management systems, with a top-level description of how one would build electronics around a battery for a car. Each chapter ends with questions and answers for readers to check their understanding of the book material.

By the end of the book, I had a hearty respect for the mathematical and modeling abilities of Rahn and Wang, but I did not have a solid feeling about how to use their methods in a simple hybrid system. For a practical prototype, much simpler models can be developed based on experimental polarization curves incorporated into relatively straightforward mathematical models. I also did not have a sense of how to use the modeling in the event that something went wrong with the battery - that is, it is unclear how to use the models to improve the longevity of the batteries or to make them safer. There is also no indication of how expensive the computational effort would be for running such detailed models.

A more accurate title for the book might be Battery Modeling for Hybrid Electric Vehicles as there is little practical systems engineering in the book. The detailed battery models would be very useful to people studying controls, and the methodology would be relevant to other electrochemical systems. The questions at the end of each chapter make it ideal for a graduate-level engineering controls class. The book would also be useful to battery materials scientists who are curious about how controls engineers work with their batteries.

Reviewer: Karen Swider Lyons researches fuel cell and battery materials and their integration into naval systems in Alexandria, Va., USA.

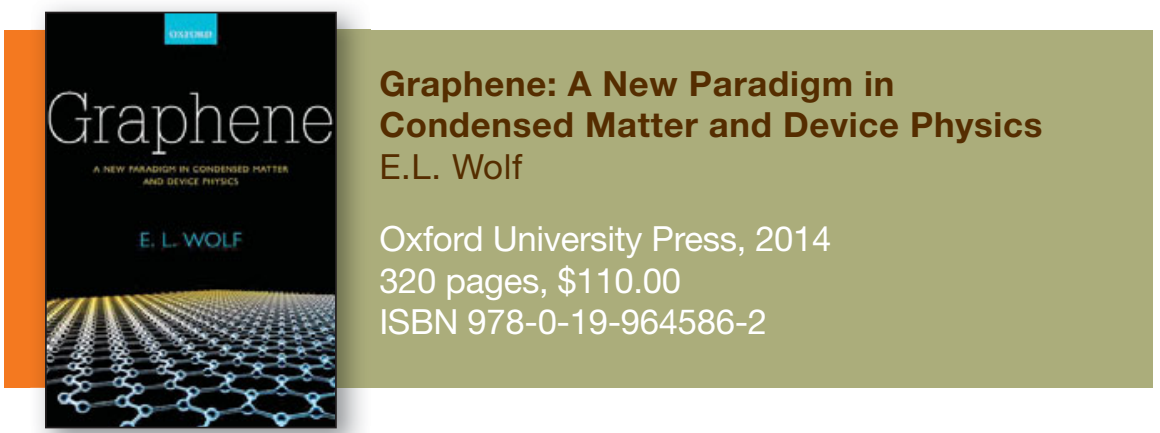

Graphene has attracted significant $\checkmark$ attention in the scientific community in recent years because of its exceptional physical properties. The 2010 Nobel Prize in Physics was awarded to Sir Andre Geim and Sir Konstantin Novoselov "for groundbreaking experiments regarding the two-dimensional material graphene." There has been an explosion in research and corresponding rapid increase in scientific publications in this area in the last decade. Given the diversity and multitude of publication sources and content, there is a need for books that will distill this information and present it in a cogent form useful for researchers. This book by Wolf serves this purpose admirably and is recommended for students and researchers who have a basic understanding of quantum mechanics and solid-state physics.

The book discusses how the structure of graphene results in the exceptional physical properties from a fundamental physics perspective. The technical content of the book begins with the description of 2D electron behavior in systems such as liquid helium and semiconductor heterojunctions (chapter 2). In the third chapter, different forms of carbon and its molecular compounds are discussed from the perspective of quantum mechanics; namely, starting from the Schrödinger equation for the carbon atom. Chapter 3 serves as a useful foundation for chapter 4 , which deals with the electronic band structure of graphene. Chapter 5 describes different methods used to synthesize graphene. Chapter 6 provides a brief overview of some of the optical, electrical, and thermal characterization techniques of this material. The mechanical and electrical properties of graphene are discussed in chapter 7 , and chapter 8 examines graphene's physical properties that are not well understood. Potential applications of graphene in devices, interconnects, Li-ion batteries, and hydrogen storage are presented in chapter 9 .

In summary, the book is a well-written and concise introduction to the structure, synthesis, properties, and applications of graphene.

Reviewer: Nirmal Govindaraju is a research assistant professor at Oklahoma State University, USA. 


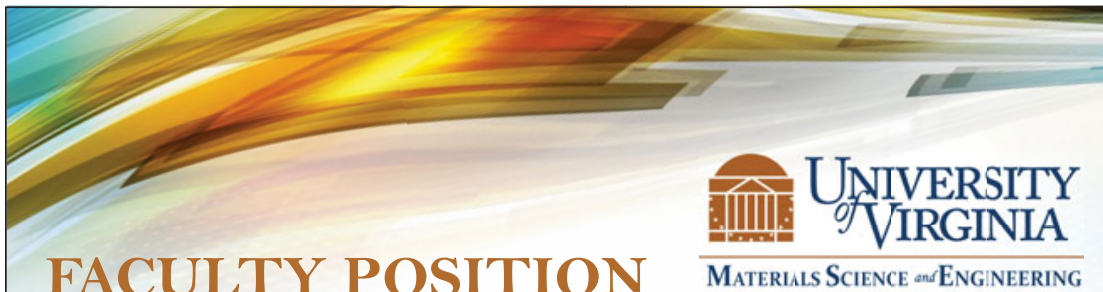

The Department of Materials Science and Engineering at the University of Virginia solicits outstanding applicants for a tenure-track faculty position at the assistant professor rank. Opportunities exist to develop state-of-the-art capabilities for independent research, experimental or theoretical/computational, for interdisciplinary collaborations with existing faculty members, and to initiate new research initiatives focused on electronic, magnetic/spintronic, or optical nanomaterials

The successful candidate will possess a doctoral degree in either materials science and engineering or a closely related field. The successful candidate will demonstrate the ability to develop a world-class, sponsored-research program centered on MS and $\mathrm{PhD}$ students and have a strong commitment to excellence in teaching at the undergraduate and graduate levels in materials science and engineering. We seek a candidate who will initiate new areas of research and teaching in the nanoscale synthesis, characterization, and/or properties of functional and multifunctional materials relevant to applications including, but not limited to, logic, energy, sensing or photonics. Competitive candidates will enhance departmental strengths through research collaborations with existing MSE faculty members, and contribute broadly across disciplines in the School of Engineering and Applied Science.
Applications must be submitted on-line through JOBS@UVA at https://jobs.virginia.edu and reference posting number 0614748 . The application closing date is January 16 , 2015. Qualified candidates are required to include a letter of application, a detailed curriculum vitae, a statement of research plans, teaching philosophy and interests as well as contact information for at least three references. The anticipated starting date for this position is August 2015.

For additional information about the position, please contact Prof. Haydn Wadley at hnw4z@virginia.edu. For questions regarding the application process, please contact Jeannie Reese at jsv7u@virginia.edu.

The University of Virginia is an equal opportunity/affirmative action employer committed to developing diversity in faculty and welcomes applications from women, minorities, veterans, and persons with disabilities.

$$
\text { - }
$$

\section{Materials Science and Engineering}
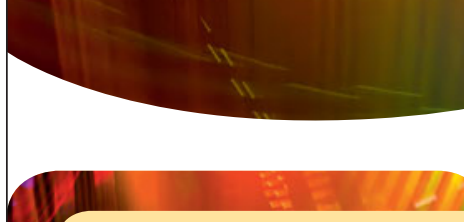

TO APPLY:

Applicants should send a curriculum vitae, statements of research plans and teaching interests and philosophy leach statement not to exceed 3 pages), and a list of at least three references (with telephone numbers and email addresses) by email as a single file in PDF format to: materials_search@seas.wustl.edu. Applications will be accepted at any time, and will be considered until the position is filled; evaluation will begin by October 15, 2014

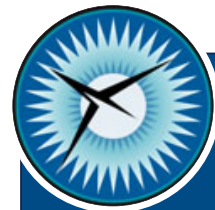

RESEARCH SCHOLAR

\section{National Institute of Aerospace}

The National Institute of Aerospace (NIA), located near NASA Langley, has an opening for a postdoctora Research Scholar to work in the general area of damage mechanics of metallic materials. Successful candidate will develop and apply characterization methods to examine the initiation and evolution of damage processes in structural metallic materials under monotonic and cyclic loading. This work is focused on quantifying the effects of microstructure on damage processes; will work independently and in a multi-disciplinary team to develop multi-scale damage models to guide the design of new more damage tolerant aerospace materials. PhD degree in engineering or physical sciences; experienced with Electron Backscattered Diffraction (EBSD) electron microscopy, crystallography and microscopy and physical metallurgy of structural metallic materials.

NIA ranked 12th in Top 50 Best Non-Profit Companies to work for in the U.S.!

For more information and to apply, go to NIA's career opportunities site at www.nianet.org.

NIA is an equal opportunity/affirmative action employer. Women, members of minority groups, individuals with disabilities, and veterans are encouraged to apply. 